\title{
Réflexions autour du projet de bibliographie des éditions lyonnaises du seizième siècle (BEL16)*
}

\author{
RAPHAËLE MOUREN \\ Université de Lyon-École nationale supérieure des sciences \\ de l'information et des bibliothèques
}

In November 2007, l'École nationale supérieure des sciences de l'information et des bibliothèques-France's national school for information and librarianshiplaunched an ambitious project, following William Kemp's proposal: establishing, in electronic form, an exhaustive, retrospective bibliography of books printed at Lyons during the sixteenth century. The implementation of this project was the object of numerous reflections, mostly upon the way the history of the book and the history of philology complement each other. Professional and disciplinary specificities concerned the identification of the types of users of such a base, the needs of these users, the norms regularly used, and the different levels of description considered to be necessary. This article recounts these conceptual progressions as they helped define bibliography in the twenty-first century. With precise comparisons to existing databases, and with concise and detailed definitions of methodology and issues, the author exposes the necessary decisions required of any bibliographic undertaking. Public, descriptions, corpus, standardization, and use are approached with reference to both conception and concept.

$\mathrm{E}$ n novembre 2007 était lancé à l'École nationale supérieure des sciences de l'information et des bibliothèques, sur la proposition de William Kemp, spécialiste du livre lyonnais de la première moitié du seizième siècle, le projet suivant : établir la bibliographie exhaustive rétrospective des livres imprimés à Lyon au seizième siècle, sous forme électronique.

La mise en œuvre de ce projet a fait l'objet de très nombreuses réflexions préalables. L'intérêt de ces réflexions tient principalement à la complémentarité des deux pilotes: William Kemp, bibliographe historien du livre, et l' auteur de cet article, chargée du suivi du projet à Lyon, qui suis bibliothécaire et spécialiste de 
l'histoire de la philologie et de l'Humanisme. Elles nous ont permis d'identifier les spécificités disciplinaires et professionnelles qui nous réunissaient ou nous séparaient : ces dernières concernent l'identification des types d'utilisateurs d'une telle base, des besoins de ces utilisateurs, les normes habituellement utilisées, les différents niveaux de description considérés comme nécessaires.

\section{Pourquoi lancer un projet de bibliographie rétrospective exhaustive de nos jours?}

L'imprimerie à Lyon au seizième siècle a fait l'objet de plusieurs travaux bibliographiques. À la fin du dix-neuvième siècle, le grand collectionneur Henri Baudrier en a établi la bibliographie, décrivant toutes les éditions connues et proposant en outre, pour chaque imprimeur, une notice biographique plus ou moins détaillée, pour laquelle il a utilisé les documents d'archives disponibles ${ }^{1}$. Ses recherches étaient approfondies, en particulier pour dater les éditions qui ne l'étaient pas, et des illustrations accompagnaient ces dix volumes. Les notices de Baudrier présentent le contenu complet de la page de titre, en transcription diplomatique : indication des sauts de ligne, de la présence d'une marque typographique ou de décorations, etc. Il précise lorsqu'il s'agit d'une réédition corrigée ou non d'une édition antérieure (avec parfois des erreurs) et indique la présence de pièces liminaires : épîtres dédicatoires, préfaces...

Un autre projet en cours est la Bibliographie des livres imprimés à Lyon au seizième siècle, supplément au vaste projet de Répertoire bibliographique des livres imprimés en France au seizième siècle lancé il y a plus de quarante ans par la maison d'édition Koerner au sein de sa Bibliotheca bibliographica Aureliana $(B B A)^{2}$. Ce projet commercial (ce que n'était pas réellement la Bibliographie lyonnaise, mené au sein de la Société des bibliophiles de Lyon par Henri puis Julien Baudrier) est mis en œuvre par une bibliographe, Sybille Von Gültlingen, par ailleurs épouse de l'imprimeur ${ }^{3}$. Un des objectifs est de publier de manière efficace les volumes. Les types de notices y sont très variés. Certaines sont diplomatiques, semblables à celles de Baudrier, mais au-delà de la description de la page de titre, on y trouve peu d'indications complémentaires : pas d'indication de réédition, ni de description des pièces liminaires. D'autres notices sont plus succinctes et même tronquées : il manque parfois des mots du titre, par exemple le nom de l'auteur décliné dans un titre latin, qui est dans ces cas ajouté en vedette. Aucune information n'est ajoutée si elle ne se 
trouve pas dans le titre, comme le nom de l'éditeur scientifique de l'ouvrage s'il n’apparaît que dans l'épître dédicatoire. Les noms d'auteurs sont modernisés mais non normalisés, avec parfois un peu de fantaisie. Mais il faut souligner que la normalisation des noms propres anciens est particulièrement difficile et lorsque l'entreprise a été lancée, les grands programmes d'autorités n'existaient pas ou n'étaient pas encore disponibles : celui de la Bibliothèque nationale de France (qui inclut désormais les notices des imprimeurs-libraires parisiens publiées par la bibliothèque ainsi que celles d'autres outils bibliographiques comme ceux de Quérard ou Antoine-Alexandre Barbier ${ }^{4}$ ) ou le Viaf (Fichier d'autorités international virtuel), lancé par OCLC avec la participation de bibliothèques nationales et spécialisées du monde entier 5 .

Des publications plus spécialisées ont aussi été réalisées, comme celle d'Alfred Cartier consacrée aux imprimeurs de Tournes ${ }^{6}$, ou la bibliographie d'Étienne Dolet de Claude Longeon, publiée en $1980^{7}$. Tous ces projets sont conçus et réalisés sous forme imprimée. Ce sont des projets bibliographiques et non pas catalographiques. Rien n'indique généralement à partir de quel exemplaire a été établie la notice ; les localisations sont succinctes et considérées comme secondaires. Elles se limitent au nom des établissements conservant un exemplaire, parfois accompagné de la cote, mais la plupart du temps aucune information n'est donnée sur l'exemplaire lui-même, comme les particularités d'état, de provenance, de reliure, annotations...

Le projet de bibliographie électronique du livre lyonnais au seizième siècle (BEL16) n'est pas isolé. Diverses entreprises de bibliographie exhaustive rétrospective ont été lancées en Europe, généralement au niveau national ou interrégional, soit à partir de projets commencés depuis longtemps sous forme imprimée puis repris et continués sous forme informatique, soit directement sous la forme de base de données. En Allemagne, les projets VD16 et VD17, consacrés au seizième et au dix-septième siècle, sont placés sous la responsabilité de la Bayerische Staatsbibliothek de Munich ${ }^{8}$; le projet italien Edit-16 est conduit à Rome par l'Istituto centrale per il catalogo unico (Iccu) ${ }^{9}$. Jean-François Gilmont a mené à bien le catalogue des ouvrages imprimés à Genève, Lausanne et Neuchâtel aux quinzième et seizième siècles, GLN 15-16 ${ }^{10}$. Ajoutons enfin le Short Title Catalogue Netherlands (STCN) terminé à la fin de l'année 2009, qui couvre la période $1540-1800^{11}$. 
Ces projets ont retenu généralement le partage géographique et linguistique qui était déjà celui des Short Title Catalogues (STC) de la British Library dans les années $1920^{12}$. Ils cherchent généralement à décrire les livres imprimés dans un pays, ou imprimés ailleurs mais dans la langue principale de ce pays : les livres publiés en Italie ou en italien pour Edit-16, les livres publiés en Allemagne ou en allemand pour VD16 ${ }^{13}$. Il ne faut bien entendu pas oublier les programmes qui se maintiennent encore sous forme imprimée, et au premier chef le "projet Renouard ", mené à bien par la Bibliothèque nationale de France, qui continue de front depuis les années 1960 l'Inventaire chronologique des éditions parisiennes $d u X V I^{e}$ siècle (en cours d'informatisation pour être versé dans le catalogue de la $\mathrm{BnF}$ ) et le répertoire alphabétique Imprimeurs et libraires parisiens $d u X V I^{e}$ siècle $e^{14}$.

Le projet le plus récent est celui de la University of St. Andrews, qui après avoir réalisé pendant dix ans la bibliographie des livres imprimés en France au seizième siècle en langue vernaculaire (French Vernacular Books), publiée sous forme imprimée et abrégée en $2007^{15}$, a élargi ce projet à la description de l'ensemble de la production européenne au seizième siècle, publiée en partie sous forme imprimée mais ouverte en 2011 sous sa forme électronique ${ }^{16}$ : ce projet, le Universal Short Title Catalogue Project, est basé sur la collaboration avec une partie des projets déjà existants, qu'il vise à fédérer et compléter ${ }^{17}$. Le projet lyonnais s'intègre donc dans une dynamique qui, à l'échelle européenne, tend à offrir la description de tous les livres publiés au seizième siècle en Europe.

\section{Quels objectifs?}

Lancer aujourd'hui, une nouvelle fois, une bibliographie des éditions lyonnaises du seizième siècle, c'est donc avoir l'ambition d'apporter de nouvelles connaissances : mais lesquelles, et comment?

Premièrement, cette bibliographie est établie à partir des moyens informatiques disponibles. Ceux-ci nous offrent de nouvelles possibilités : l'enrichissement et la mise à jour toujours possibles, les modes d'accès et d'interrogation multipliés, la liberté de donner des informations et des illustrations sans limite technique ou financière. Les catalogues de bibliothèques en ligne signalent aujourd'hui un grand nombre d'éditions inconnues des bibliographies de Baudrier et Gültlingen. Il suffit en effet de lancer une recherche dans les catalogues et métacatalogues et d'être patient; on moissonne 
ainsi pour chaque imprimeur des livres inconnus des bibliographies, décrits pourtant par les bibliothèques qui les conservent ; ces nouvelles localisations, qui nécessitent la consultation régulière des catalogues des bibliothèques (car ceux-ci s'enrichissent chaque jour) demandent bien entendu des vérifications avant intégration, ne serait-ce quà cause du mode de conversion rétrospective utilisé par les bibliothèques - en particulier les bibliothèques françaises pour informatiser leurs catalogues ${ }^{18}$.

Deuxièmement, les outils bibliographiques ne sont pas moins utiles, et même le sont davantage, avec le développement des entreprises de reproduction in extenso de l'ensemble des textes et documents des plus grandes et plus importantes collections mondiales. En effet, la bibliographie, sous sa forme électronique, devrait pouvoir constituer à terme le catalogue de ces immenses ensembles numérisés, organisant ainsi au mieux l'information. C'est en tout cas un élément à prendre en compte dès la conception d'un tel outil, en particulier pour des questions techniques. En quelque sorte, les projets de bibliographie peuvent proposer une indexation de qualité et contrôlée de ces projets de numérisation et en constituer les métadonnées - à condition bien entendu de permettre à l'utilisateur de faire le lien entre les deux et d'utiliser des moyens d'identification compatibles. Ils pourront permettre ainsi de corriger une faiblesse des bibliothèques numériques de grande ampleur ; ils offriront des informations complètes à l'utilisateur des bibliothèques numériques, et par là même multiplieront les recherches possibles.

Troisièmement (mais pas en ordre d'importance), l'objectif est de permettre l'identification des nombreuses éditions encore anonymes, dont on ignore le lieu d'édition et le nom de l'imprimeur. Pour cela, l'idée est de mettre à disposition des reproductions du matériel typographique utilisé, permettant ainsi d'identifier aussi des livres incomplets (en particulier lorsqu'un exemplaire a perdu sa page de titre).

Cet outil se veut donc utile pour les livres aisés à décrire comme pour les plus complexes, mais aussi pour identifier les doublons réels ou faux, les émissions et éditions partagées, très nombreuses à Lyon au seizième siècle et parfois fort difficiles à identifier. Il est aussi conçu pour être utile à des utilisateurs spécialistes de diverses disciplines, et pas seulement aux bibliographes et aux historiens du livre : aussi bien à ceux qui s'intéressent à une édition pour des raisons variées quà ceux qui étudient des exemplaires spécifiques. Il s’agit bien 
là de donner un meilleur accès à ce patrimoine technique et intellectuel, fruit d'un important travail technique et scientifique.

Bien évidemment, il est manifeste dès l'abord que le choix de dates comme « le seizième siècle » ne correspond pas à la réalité de l'histoire de l'imprimerie, et que nombre d'imprimeurs du seizième siècle sont actifs au siècle précédent. Mais la description des incunables est complexe, et plusieurs projets mondiaux couvrent très bien le quinzième siècle, que ce soit le Gesamtkatalog der Wiegendrucke (GW, Union catalogue of incunabula) ou l'Incunabula ShortTitle Catalogue anglais (ISTC) ${ }^{19}$. Ils complètent les catalogues d'incunables établis par les bibliothèques, qui décrivent souvent de façon détaillée et précise les exemplaires. Il existe en outre de nombreux outils permettant d'identifier un incunable. Nécessairement, des notices d'imprimés édités au quinzième ou au dix-septième siècle seront présentes dans la bibliographie lyonnaise, car on s'attachera à décrire l'intégralité de la production lyonnaise de chaque imprimeur. Mais notre priorité n'est pas de contrôler les travaux déjà existants, simplement de les compléter : dans le cas des incunables, l'idéal serait de pouvoir donner un lien permanent vers la notice correspondante du GW ou de l'ISTC.

\section{Une description enrichie}

Dès ces premières réflexions, il apparaît que notre ambition est de donner un très grand nombre d'informations ordonnées et classées. Aujourd'hui, la recherche d'informations à l'intérieur de bases de données, moteurs de recherche, métacatalogues et catalogues collectifs est devenue une démarche courante pour les chercheurs. Google par exemple permet de faire un certain nombre de recherches, dans sa propre bibliothèque numérique comme dans les autres bibliothèques numériques, et, de plus en plus, directement dans les catalogues des bibliothèques - si tant est que les métadonnées permettant le référencement sont bien présentes - par auteur ou titre essentiellement mais évidemment aussi par mot présent dans l'ensemble du texte. Les catalogues de bibliothèque en ligne multiplient les accès en offrant des notices dont certaines sont régulièrement améliorées. Les métacatalogues (Karslruher Virtueller 
Katalog, World $\operatorname{cat}^{20}$ ) permettent désormais de mener simultanément une recherche dans des catalogues de bibliothèques et de bibliothèques numériques ainsi que dans des bibliographies spécialisées en ligne. Où est donc la place de la bibliographie ? Assurément dans le soin donné à la description, et lorsque c'est nécessaire, dans le travail pointu de vérification mis en œuvre, y compris de mise à jour des connaissances grâce au repérage des nouveaux exemplaires signalés et à l'utilisation des travaux de recherche les plus récents (par le dépouillement de la bibliographie mais aussi par la participation active de chercheurs au projet) ; elle corrige ainsi les erreurs d'attribution recopiées et répétées de notice en notice depuis parfois plusieurs centaines d'années, qui deviennent ainsi des références indiscutables en apparaissant sur un écran, et qui font parfois faire à notre connaissance du livre ancien un bond en arrière de plusieurs dizaines d'années, gommant les progrès du siècle dernier. Elles complètent les informations incomplètes réunies à partir d'exemplaires incomplets.

La place de la bibliographie est aussi dans une organisation logique de l'information - les métadonnées chères aux informaticiens -, selon des critères adaptés à de nombreux types de recherche et non pas seulement centrés sur un petit nombre d'entre eux : auteur, titre et sujet ne sont pas toujours les critères de recherche les plus pertinents. Sa place est enfin dans la mise à disposition d'informations disparates intellectuellement réunies : notice descriptive, images, références bibliographiques... La bibliographie a donc une exigence de recherche d'information qui va au-delà de la description de ce que l'on voit simplement en ouvrant un livre pour en copier la page de titre.

\section{Après les « short title catalogues "}

La base de ce projet est de faire mieux que ce qui existe, ou plus exactement et plus modestement - d'offrir une documentation plus complète et moins dispersée que celle dont on dispose pour l'instant. L'établissement de catalogues de bibliothèques et de bibliographies a connu plusieurs étapes ${ }^{21}$. La différence principale entre les divers projets de catalogues ou bibliographies du siècle dernier tient dans la forme des notices : les notices abrégées (« shorttitle » en anglais) donnent un nombre réduit d'informations, ce qui permet essentiellement de les rédiger beaucoup plus rapidement. Des notices complètes apportent des informations supplémentaires : la rédaction demande plus de 
temps, même si des outils de travail de plus en plus perfectionnés peuvent aider désormais le bibliographe ${ }^{22}$. On verra plus loin que ces informations supplémentaires, que l'on ne trouve pas sur la page de titre mais à l'intérieur du livre, peuvent apporter de nombreuses précisions sur l'édition elle-même et sont indispensables à certains types de recherches.

La forme de description abrégée a été retenue pour établir des bibliographies, ce qui permet l'efficacité et la mise à disposition rapide de l'information : c'est le cas par exemple du Short-title Catalogue of Books printed in England, Scotland and Ireland, and of English Books printed abroad, 1475-1640, publié en 1926 par Gilbert Redgrave et Alfred Pollard et mis à jour à plusieurs reprises puis informatisée ${ }^{3}$. Plus récemment, c'est aussi le choix de l'Index Aureliensis, publié par fascicules depuis $1965^{24}$. Dans ce cas précis, il me semble qu'on est presque dans une réflexion qui utiliserait volontiers l'outil informatique mais doit se cantonner au papier. La première étape est le signalement de tous les livres édités au seizième siècle, sous forme abrégée. Toute notice abrégée de l'Index Aureliensis semble avoir pour vocation à être un jour complétée et peut-être corrigée par une notice complète dans un des volumes de la Bibliotheca bibliographica Aureliana.

Pour les catalogues de bibliothèques en particulier, le choix des shorttitle catalogues, des catalogues de notices abrégées, avait été fait par la British Library dans les années 1920. Il a permis la fabrication rapide d'outils de localisation de ses propres collections; ces catalogues, découpant les collections de la bibliothèque par période et par aire géographique, pouvaient même être consultés comme des bibliographies, tout simplement à cause de la très grande richesse de la British Library ${ }^{25}$.

Les catalogues constitués de notices abrégées permettent de vérifier qu’une édition existe, ou qu'elle est réputée exister lorsque les localisations ne sont pas données ${ }^{26}$. Mais dans la plupart des cas, elles donnent trop peu d'informations au chercheur pour pouvoir être utilisées sans être complétées par une notice plus complète ; elles permettent rarement de savoir si un livre est une réédition d'une édition précédente, s'il s'agit d'une édition en collaboration... Leur consultation doit nécessairement être suivie de contrôles, soit sur d'autres travaux s'ils existent, soit sur l'ouvrage lui-même - option dont il ne faut pas oublier l'existence : les nouveaux outils ne font pas disparaître le travail du chercheur en bibliothèque, livre en main... 
Avec l'évolution rapide des catalogues, due à la fois à leur informatisation et à leur mise à disposition plus large par le biais d'Internet, à la conversion rétrospective de grande ampleur qui a été menée (tous les fichiers papier ont été informatisés, à la British Library, à la Bibliothèque nationale de France, mais pas partout, attention !), la notice abrégée n'est plus forcément une étape nécessaire sur la route de la constitution d'un catalogue.

Intégrer dans un catalogue ou dans une bibliographie des notices abrégées aujourd'hui, c'est frustrer des chercheurs définitivement habitués à d'autres formats grâce aux catalogues informatisés des bibliothèques, qui donnent immédiatement une information plus complète mais la donnent aussi parfois corrigée et mise à jour, y compris pour les notices de livres du seizième siècle : c'est en tout cas ce que fait la Bibliothèque nationale de France, comme ont certainement pu le constater ceux qui ont eu à consulter à plusieurs années d'intervalle la notice du même livre. Mais ce choix, celui des notices abrégées, peut s'avérer nécessaire et même la solution de bon sens, lorsque l'objectif est de mener à bien la description du contenu d'une bibliothèque, en fonction de nombreux facteurs ; le bibliothécaire doit prendre en compte l'intérêt des collections dont il a la charge, le moyens humains, techniques et financiers dont il dispose, les publics intéressés... ${ }^{27}$

Le temps de la notice abrégée semble donc passé pour les bibliographies comme pour les catalogues. C'est un choix qui continue pourtant à être fait, comme c'est le cas pour les notices de la base de données Early English Books Online (EEBO), projet privé accessible par abonnement; il est vrai que cette base intègre les notices du Short-Title Catalog of Books Printed in England. Cette base de données permet des recherches multiples, la conservation de l'historique des recherches, et ajoute aux notices des images, en particulier des reproductions des pages de titre. Les notices elles-mêmes reproduisent simplement la page de titre, tronquant parfois le titre s'il est trop long $^{28}$. Il en est de même pour l'English Short Title Catalogue, lancé en 1977 pour compléter les STC imprimés en couvrant le dix-huitième siècle et qui a pour objectif de décrire l'intégralité de la production imprimée des pays de langue anglaise (y compris les fausses adresses) et tous les livres publiés en anglais ${ }^{29}$.

Cette forme a également été retenue récemment par la University of St.Andrews et son éditeur Brill. La sortie à la fin de l'année 2007 des deux volumes French Vernacular Books : Books Published in the French Language before $1601^{30}$ a entraîné des réactions contrastées de la part des chercheurs 
intéressés par le domaine, spécialistes de bibliographie, de littérature française du seizième siècle ou de l'histoire de l'édition : ils y ont fait des découvertes nouvelles, mais ont exprimé une intense frustration, et certains ont relevé la réapparition d'éditions fantômes pourtant identifiées, ce qui nécessite des vérifications avant utilisation des informations proposées dans ces volumes. Il faut toutefois préciser que ces deux volumes sont l'abrégé de la base de données du même nom, qu'il était alors techniquement impossible de rendre disponible en ligne, et dont les notices sont bien plus riches que celles qui ont été extraites pour l'édition ${ }^{31}$. Preuve, s'il en fallait, que si la forme imprimée se justifie encore dans de nombreuses publications, elle est un choix lourd de conséquences dans le domaine spécifique de la bibliographie : dans le cas de French Vernacular Books, les nombreuses corrections relevées par les spécialistes dès la sortie du livre ne l'ont été que parce que ceux-ci savent que, derrière le papier, il existe une base électronique dans laquelle les corrections pourront être intégrées ; il pourrait difficilement en être de même pour les volumes actuellement en vente chez l'éditeur, sauf à imaginer de rébarbatives listes de corrections dont l'efficacité est réduite.

Cette expérience montre quoiqu'il en soit les difficultés que l’on rencontre en tentant d'établir une bibliographie rétrospective fiable, sur des critères scientifiques : certaines sources demandent une vérification systématique, en particulier les catalogues de bibliothèques dont certaines notices mal rédigées inventent une édition qui n'existe pas au lieu de rattacher l'exemplaire à une édition bien identifiée.

Les critiques portées sur cet ouvrage par les spécialistes, les erreurs qu'ils ont relevées montrent qu'ils attendent d'un tel projet la recherche de l'excellence, la réflexion sur ses objectifs et ses usages et l'utilisation des travaux de recherche existants, même s'ils sont parfois difficiles à trouver. Il faut donc prendre en compte avec beaucoup d'attention cette attente et ces besoins, par exemple en organisant la possibilité pour les spécialistes de participer au projet en faisant connaître directement leurs découvertes. Cette collaboration au niveau international est aussi un moyen d'enrichissement de la base BEL16, qui réunit déjà plusieurs spécialistes. 


\section{Description ou reproduction?}

Pour tempérer la tentation de passer de la notice abrégée à une notice qui donnerait le plus grand nombre possible d'informations, au risque de constituer trop lentement la bibliographie pour en venir à bout, il faut prendre en compte les outils complémentaires existants ou en cours de constitution. L'enrichissement exponentiel des réservoirs d'images, et tout particulièrement de reproductions de livres anciens, entraine une nécessaire réflexion sur le contenu des notices bibliographiques de livres anciens. Faut-il proposer une transcription diplomatique de la page de titre, reproduisant chaque détail, indiquant quelles lettres sont en capitales, où s'arrête chaque ligne, la taille des caractères pour chaque ligne? N'est-il pas bien plus rapide, et bien plus efficace, de proposer une photographie de la page de titre, et de s'attacher alors plutôt à donner les informations permettant d'identifier ce livre et surtout, y ajouter les informations qui ne sont pas présentes sur la page de titre ? Établir en effet une notice décrivant en détail le contenu et cherchant à faire comprendre l'apparence de la page de titre demande du temps, et reste tout compte fait peu satisfaisant : le seul moyen de savoir exactement à quoi ressemble cette page de titre n'est-il pas de la voir ${ }^{32}$ ?

\section{Questions techniques et normalisation}

\section{Logiciels et outils informatiques}

Une des premières réflexions à mener concerne les difficultés et les dangers d'une telle initiative, y compris les pièges techniques et scientifiques.

Parmi les projets existants, certains sont menés par des bibliothécaires : c'est le cas des projets allemands VD16 et VD17, du projet italien Edit-16 ; d'autres le sont par des bibliographes, comme le Universal Short Title Catalogue Project écossais ou GLN 15-16 de Jean-François Gilmont, qui est à la fois bibliographe et bibliothécaire. Ces projets sont proches dans leurs objectifs, mais se différencient bien souvent par leur envergure, qui fait pencher les uns du côté du catalogue collectif thématique, les autres, du côté de la bibliographie rétrospective exhaustive. Ainsi, le projet italien Edit-16 vise à recenser les livres publiés en Italie ou en italien conservés dans les bibliothèques participant au projet (signalons qu'aux bibliothèques italiennes s'ajoutent depuis peu 
des établissements étrangers). Les notices et les formes normalisées des noms sont reprises et améliorées par rapport au catalogue collectif italien, le Servizio bibliotecario nazionale, en particulier l'identification sous leur nom réel des acteurs du livre, qui toutefois ne se fondent pas dans un même fichier d'autorités ${ }^{33}$; mais la limite de la participation aux bibliothèques d'un territoire spécifique, malgré l'entrée récente de bibliothèques étrangères (dont la Bibliothèque nationale de France) réduit son ampleur. On peut penser, sans doute, que la richesse des bibliothèques italiennes entraîne bien peu de manques dans ce projet : mais même peu nombreux, il existe certainement des exemplaires uniques d'éditions italiennes du seizième siècle en dehors du territoire actuel du pays. Cela a aussi une incidence sur la question de la description des exemplaires connus et limite fortement l'intérêt d'un tel outil pour des utilisateurs comme les spécialistes de l'histoire de la culture, l'histoire des bibliothèques ou de la lecture, l'histoire économique et sociale du livre, etc. Les limites sont les mêmes pour VD16, qui n'est enrichi que par les collections des bibliothèques allemandes participant au projet : cela exclut les livres conservés en dehors du territoire de l'Allemagne actuelle, ainsi que les livres dont aucun exemplaire n'est localisé aujourd'hui mais que des sources sûres nous indiquent qu'ils ont existé.

Pour qui observe de près et utilise ces outils, il est manifeste que bibliothécaires et bibliographes travaillent différemment, ne communiquent pas entre eux, et obtiennent des résultats parfois fort différents.

Pour William Kemp, GLN15-16 est actuellement « la plus perfectionnée et la plus avancée des bibliographies web portant sur le seizième siècle». Je pense pour ma part que c'est certainement la plus perfectionnée et la plus avancée, ne serait-ce que parce que le plus grand spécialiste actuel du domaine a vu luimême toutes les éditions et les a décrites parfaitement, mais je pense aussi que ce projet sous-utilise les possibilités nouvelles offertes par l'informatique. En l'état actuel de la base de données, celle-ci donne l'impression fausse d'avoir été conçue de la même façon que s'il s'était agi de préparer un volume imprimé, ce qui n'est en réalité pas le cas. Il est certain que le projet a pâti des limites techniques du logiciel utilisé, la base de données utilisée pour saisir les notices (Filemaker Pro) ayant été directement mise en ligne.

Certains des choix dans la conception de cette base de données peuvent surprendre le bibliothécaire : celui, par exemple, d'attribuer un numéro à toute notice nouvelle. Le bibliothécaire a en effet l'habitude de laisser son logiciel de 
catalogage attribuer automatiquement un numéro de notice. D’autres choix, nés de la volonté d'efficacité, donnent des informations incomplètes : ainsi, dans le cas d'une édition grecque comme l'édition d'Eschyle publiée à Genève en 1557 par Henri Estienne, il est indiqué que la langue et l'écriture du livre est le grec. Or, la préface et la postface sont écrites en latin... La mention de langue indique donc, non pas la seule langue utilisée dans le livre, mais la langue principale : précision sans doute inutile pour les bibliographes spécialisés qui consultent la base de données, mais indispensable pour les utilisateurs moins aguerris. Tous les exemplaires identifiés sont signalés, celui qui a été consulté est indiqué, mais aucune autre information sur les exemplaires n'est donnée, en particulier sur leur histoire et leur provenance. Un chercheur travaillant sur la réception et à l'utilisation de cette édition, ou bien encore sur l'histoire des collections, ne trouve pas grand chose pour l'aider dans cette bibliographie, dont il doit compléter la constitution par de fastidieuses recherches dans les catalogues des bibliothèques conservant un exemplaire. Il faut toutefois rappeler que malheureusement, en l'absence la plupart du temps de permaliens sur les notices des catalogues de bibliothèque, il est impossible de mettre simplement un lien vers la notice dans le catalogue de la bibliothèque.

Les projets menés par des bibliothécaires utilisent assez naturellement les logiciels dédiés pour les catalogues de bibliothèque (appelés systèmes intégrés de gestion de bibliothèque, SIGB, ou bien systèmes d'information, SI) : VD16 et VD17 utilisent ainsi le logiciel Aleph, logiciel qui permet le plus facilement l'utilisation de caractères non latins. Les logiciels utilisés pour les catalogues sont généralement propriétaires et très chers ; une évolution récente et rapide voit toutefois la progression de l'utilisation de logiciels libres tels que Koha. Ils sont aujourd'hui largement paramétrables et extrêmement performants.

Les bibliographes, de leur côté, sont plutôt tentés, pour constituer leurs bases de données par les outils généralistes de bases de données : Microsoft Access avait été choisi par le projet French Vernacular Books de la University of St. Andrews, ce qui les a conduits dans une impasse. La base de données a été lancée il y a plus de quinze ans, en utilisant un outil bureautique de base grand public. Il a longtemps été impossible de la transférer dans un autre logiciel afin de pouvoir la mettre en ligne, ce qui explique sans doute la parution récente d'une forme papier de cette bibliographie. Le problème est réglé et la structure informatique permettant de mettre en ligne ce corpus est désormais développée ; en revanche, le choix technique retenu demandera certainement 
une réflexion supplémentaire sur la sauvegarde des données à long terme. Jean-François Gilmont a choisi pour GLN15-16 un logiciel du même type, c'est-à-dire un logiciel de bases de données grand public, FilemakerPro, qui a l'avantage d'être transférable dans le format XML (ce qui permet de transférer et conserver les données), et de pouvoir être mis en ligne. Le résultat est toutefois peu satisfaisant en comparaison avec la richesse de cette bibliographie et à la souplesse à laquelle les chercheurs ont été habitués par certains catalogues de bibliothèques.

Notons l'existence d'un logiciel qui semble réunir bibliothécaires et bibliographes, mais qui n'est utilisé pour l'instant que très peu, aux États-Unis et en Grande-Bretagne essentiellement ; il s'agit du moteur libre Cheschire 3, utilisé en particulier pour le l'ESTC ${ }^{34}$.

Il est donc indispensable, en lançant un projet de ce type, de réfléchir aussi bien aux questions scientifiques et intellectuelles qu'à ses aspects techniques, pour éviter de rencontrer par la suite des obstacles insurmontables et pour assurer la pérennité de la base de données et de sa consultation.

\section{Structure des notices}

On observe des différences entre les divers projets actuellement en cours dans le choix de la forme même des notices : ces différences s'expliquent par des traditions bibliographiques différentes, entre bibliographes d'un côté et bibliothécaires de l'autre, mais aussi par le manque de connaissances de bibliographes improvisés, attirés par Internet, troisième catégorie de concepteurs de bases de données bibliographiques.

Les bibliothécaires ont depuis longtemps concentré leurs efforts sur la normalisation ; on le voit déjà dans les prescriptions données en France par les révolutionnaires aux responsables des dépôts littéraires, où furent réunies les bibliothèques des aristocrates, des ordres religieux et des corps constitués saisis par la Révolution. Ils imposaient le catalogage immédiat, et faisaient des recommandations sur les informations à reporter, et même aussi sur des dimensions normalisées des fiches : ils préconisaient en effet l'usage généralisé du dos des cartes à jouer ${ }^{35}$. Au dix-neuvième siècle les circulaires répétées du ministère de tutelle aux bibliothèques conservant ces collections anciennes s'arrêtent parfois sur la forme que doit prendre la description des livres, mais il règne encore une grande disparité dans les fiches de cette époque. C'est à partir 
de la fin du dix-neuvième siècle, en particulier avec le projet de bibliographie universelle de Paul Otlet et Henri Lafontaine, que la normalisation s'accélère ${ }^{36}$.

Les bibliothécaires s'appuient depuis une quarantaine d'années sur un ensemble de recommandations de l'International Federation of Library Associations and Institutions (IFLA), l'International Standard Bibliographic Description (ISBD). Cette norme, fondée sur les Principes internationaux de catalogage de $1961^{37}$, a d'abord été déclinée en fonction des types d'objets à cataloguer : I'ISBD (A) concernait le livre ancien et a fait l'objet d'une adaptation française par l'agence française de normalisation, l'Afnor, en cours de révision. Il existait aussi l'ISBD(PM) pour la musique imprimée, l'ISBD (CM) pour les cartes et plans, l'ISBD(NBM) pour les autres supports. Ces normes sont aujourd'hui remplacées par l'ISBD intégré, publié en $2011^{38}$. Cette structure rigide est complétée par des zones de notes, dont on peut, dans le cadre d'une entreprise bibliographique, envisager l'adaptation à une demande précise de description qui ne serait pas prévue. Elle n'est toutefois pas assez rigide pour l'outil informatique, laissant une marge dans les choix de catalogage qui gêne considérablement les possibilités d'interrogation des catalogues. L'ISBD est aujourd'hui concurrencée par la norme RDA, Ressources : description et accès (Ressource Description and Access), conforme aux nouveaux Principes internationaux de catalogage publiés en 2008 par l'IFLA et davantage orientés vers les usagers ${ }^{39}$. Cette nouvelle norme, qui concurrence l'ISBD, a fait l'objet de tests à large échelle et sera adoptée par les bibliothèques des États-Unis d'Amérique dès 2012.

Les bibliothécaires s'appuient aussi sur le format de description Marc, dont il existe un certain nombre de variantes suivant les pays : Marc21 aux États-Unis, Intermarc en particulier à la Bibliothèque nationale de France, Unimarc dans les autres bibliothèques françaises ${ }^{40}$. Il s' agit d'une normalisation des champs de description, les mêmes informations étant toujours présentes dans les mêmes champs ou sous-champs, ce qui permet une récupération exhaustive des données en cas de changement de système et permet aux bibliothécaires d'avoir des habitudes communes. C'est l'utilisation de Marc qui permet la mise en place de catalogues collectifs de bonne qualité comme le catalogue collectif des bibliothèques universitaires françaises, le Sudoc. Les bibliothécaires se tournent aujourd'hui de plus en plus vers les formats XML, utilisables et transférables sans logiciel spécifique, ainsi que Dublin Core, utilisés en particulier pour structurer les notices des bibliothèques numériques. 
Les bibliographes ont une longue tradition de description des livres, en particulier des livres imprimés. Les méthodes de description des libraires parisiens, mises en œuvre à la fin de l'Ancien Régime dans les catalogues de ventes de bibliothèques privées, ont atteint leur apogée avec le Manuel $d u$ libraire de Brunet ou les bibliographies consacrées par Antoine-Augustin Renouard aux imprimeurs Manuce et Estienne ${ }^{41}$. On peut rappeler aussi des exemples étrangers, comme les annales des imprimeurs Giunta établies au XVIII ${ }^{e}$ siècle par le bibliothécaire florentin Angelo Maria Bandini, par ailleurs auteur de catalogues des manuscrits des bibliothèques dont il avait la charge ${ }^{42}$.

Qu'en est-il aujourd'hui ? Dans les bibliographies, la saisie est beaucoup moins normalisée qu' elle ne l'est dans les catalogues de bibliothèques. Le respect du contenu de la page de titre et sa reproduction à l'identique n'apparaissent pas comme une règle de base incontournable, alors qu' il l'est pour le bibliothécaire qui considère que la comparaison entre la page de titre d'un livre qu'il a en main et celle que décrit une notice bibliographique ou catalographique est la première étape, indispensable, de l'identification. En revanche, les bibliographes tentent rarement de normaliser à tout prix leurs notices, comme l'ont fait pendant une période les bibliothécaires lorsqu'ils utilisaient les indications de format (in-octavo, in-quarto) pour classer des ouvrages de diverses dimensions, alors même que ces livres, lorsqu'ils n'ont pas été fabriqués à l'époque artisanale, ne sauraient répondre à ces caractéristiques.

L'absence de communication entre ces deux mondes fait que les bibliographes ne connaissent pas toujours l'existence des normes et formats utilisés par les bibliothécaires, ou les maîtrisent parfois mal. Mais au-delà des différences dans la tradition de chacun de ces métiers, compensée par la précision avec laquelle les bibliographes et les bibliothécaires décrivent les livres, on observe une ignorance totale de ces questions de normalisation et de format depuis que la constitution de bases de données est devenue très prisée des chercheurs spécialistes d'autres domaines, qui décident de se tourner vers la constitution d'outils sophistiqués sans toujours chercher à connaître réellement le domaine de la bibliographie et du catalogage, ni le travail de leurs prédécesseurs. Un certain nombre de ces projets débutent donc par de longs travaux préalables destinés à établir un formulaire de description de livres anciens, travaux qui s'avèrent pourtant totalement inutiles lorsque la réflexion porte sur les champs de description communément utilisés. Les chercheurs ont 
aussi souvent du mal à différencier les questions de normes des questions de formats.

Trop souvent, on s'aperçoit que la réflexion a porté sur des aspects banals (champs de description de base), alors qu'un petit nombre d'entre eux auraient dû nécessiter du travail, de la réflexion et demander des décisions. Plus grave : nombreux sont ceux qui font mal le partage entre des outils différents qu'ils trouvent à leur disposition en ligne, en particulier entre les catalogues de bibliothèques (Bibliothèque nationale de France par exemple), les catalogues collectifs non centralisés (Catalogue collectif de France), et des «bases de données » (expression qui n'a pas de sens particulier et dont l'emploi est mal maîtrisé) constituées à partir d'un projet scientifique donné, qu'il soit bibliographique ou autre ; certains de ces outils sont d'ailleurs simplement des listes, d'autres sont perfectionnés et constituent de véritables projets bibliographiques appuyés sur des connaissances scientifiques approfondies : citons pour exemple la remarquable Bibliographie des livres scientifiques imprimés à Rome (1527-1720) établie par Laurent Pinon ${ }^{43}$.

On observe donc une compréhension souvent inappropriée de ces outils dont la qualité est nécessairement variable, en fonction de l'histoire de leur constitution. Et surtout ils sont parfois utilisés au même niveau de recherche d'information, ce qui est inquiétant - et surprenant : un outil de localisation, surtout s'il a été constitué à partir de notices établies entre 1803 et 1980, simplement recopiées au moment de leur informatisation, a en réalité peu de points communs avec un outil de description établi en utilisant les recherches les plus récentes menées dans le domaine. Ce mélange n'est pas très grave lorsqu'il est accompagné d'une très grande exigence scientifique, dans un projet maîtrisable. Il l'est davantage lorsque, comme on peut le voir dans quelques exemples du projet French Vernacular Books, il entraine la réapparition d'éditions fantômes pourtant bien identifiées (ou même pas encore identifiées comme telles), simplement parce qu'une notice de catalogue a été établie à partir d'un exemplaire incomplet - ce qui entraîne la mention [s.l.] (sine loco) ou [s.d.] (sans date) — ou par un bibliothécaire non spécialiste qui n'a pas su décrypter une page de titre trop complexe ou compléter correctement des éléments de description présents à d'autres endroits du livre. La question de la provenance des notices, de la fiabilité de leur auteur ou de la vérification qui a pu être faite sur l'exemplaire lui-même, est ici centrale. 
La normalisation toutefois, même organisée au niveau international et contrôlée par des organismes spécialisés, reste difficile : prenons l'exemple des notices d'autorité, dont la normalisation a été entreprise plus récemment. En Italie, les notices d'autorité auteurs ne sont pas toujours les mêmes dans Edit16 et dans le catalogue collectif italien, le SBN : celles d'Edit-16 sont meilleures, mais ce sont celles du SBN qui font autorité au niveau international, et ce sont donc ces dernières, même fautives, qui sont utilisées par la Bibliothèque nationale de France pour les auteurs italiens. Il s'avère que ces deux bases sont gérées séparément, ce qui est assez étrange. Dans la constitution même des notices, les utilisateurs savent bien que les bibliothécaires ont des habitudes très variées en ce qui concerne la transcription du contenu des pages de titre : comment transcrire les $u$ et $i$ des mots latins, qui s'écrivent $V$ en capitale et $j$ en fin de mot ? Faut-il ou non développer les abréviations ? Les normes, même dans leur plus récente mise à jour, peinent à guider en permanence le catalogueur.

\section{Les apports nouveaux}

Il me semble que bibliothécaires comme bibliographes tardent à s'approprier une bonne partie des possibilités offertes par l'outil informatique. Prenons comme exemple les formes d'autorité, autrefois appelées « vedettes » par les bibliothécaires. Généralement, en construisant la notice descriptive d'un livre, on ajoute un certain nombre de ce que les bibliothécaires appellent les accès contrôlés : auteurs, imprimeurs, mais aussi lieu, désormais la date... Or, compléter chaque notice en y ajoutant tous les accès contrôlés nécessaires peut être très long. Il serait plus efficace que les formes orthographiques et linguistiques différentes de chaque nom soient intégrées dans une base spécifique la première fois qu'elles apparaissent, plutôt que d'avoir, pour chaque notice, à ajouter la notice d'autorité correspondante, et faire les renvois nécessaires pour permettre les recherches. L'outil informatique peut ensuite aider à l'enrichissement des notices en proposant automatiquement la forme d'autorité correspondante. Dans le cas de l'imprimeur lyonnais Guillaume Rouillé ou Roville par exemple, onze formes différentes apparaissent sur ses livres. Il est inutile d'intégrer dans chacun de ses très nombreux livres (plusieurs milliers) la forme d'autorité principale : il sera bien plus rapide de signaler au système l'existence des onze formes différentes, qu'il utilisera ensuite pour compléter les notices mais aussi pour chaque recherche faite par un utilisateur 
sur le catalogue de cet imprimeur. Il en est de même pour les noms d'auteurs ou de villes. Ce type d'approche est davantage une réflexion d'informaticien que de bibliothécaire, qui a, lui l'habitude d'ajouter systématiquement ses accès contrôlés, en fait, sur ordinateur comme il le ferait sur papier. Il faut mettre en œuvre les moyens de faciliter et d'accélérer le travail de saisie, en abandonnant les habitudes anciennes héritées du temps de la description sur papier, mais aussi, et en même temps, les moyens d'assurer de meilleurs résultats pour les recherches.

Dans le même ordre d'idée, la différence entre le papier et l'informatique est bien celle de l'espace. Lorsqu'on rédige une bibliographie destinée à être imprimée, les considérations économiques jouent un rôle non négligeable ; il faut toujours gagner de la place. C'est ce qui explique que le projet FVB ait fait l'objet d'une publication en notices abrégées, alors que les notices complètes existent pour chacun de ces livres. Vu le prix de l'octet, la question ne se pose plus pour l'informatique. À quoi sert alors de continuer à utiliser des abréviations incompréhensibles, surtout si elles sont très nombreuses, pour les références bibliographiques ou pour les noms des établissements de conservation ? La solution, pour assurer à la fois un gain de temps pour la saisie, mais aussi, et peut-être surtout, un confort de lecture pour le lecteur qui ne serait pas un spécialiste chevronné (un spécialiste de disciplines moins habituées au livre, un chercheur débutant), n'est-elle pas d'indiquer en toutes lettres les noms des bibliothèques, les titres des références bibliographiques, en utilisant là encore, les richesses de l'outil informatique pour automatiser en partie la saisie?

Une autre solution est celle qu'a choisie par Edit-16 : une abréviation qui se résout si on place le curseur dessus. Seul inconvénient : impossible de copier automatiquement la référence, ou même de la recopier autrement que sur une feuille de papier, le curseur ne pouvant être déplacé...

\section{Quelles informations pour quelles recherches?}

Enfin, la réflexion sur la description, et en particulier sur les types d'information que l'on souhaite relever dans le livre, doit prendre en compte les besoins des chercheurs. Il faut en effet, c'est indispensable aujourd'hui, se poser la question des publics visés par ce genre d'entreprise.

Rassembler une bibliographie de ce type permet dans un premier lieu de nous faire une meilleure idée de la production du livre au seizième siècle, 
à Lyon, et de mieux connaître le travail des imprimeurs et libraires. Pour les spécialistes de l'objet livre, les apports sont importants ; la bibliographie aidera certainement à l'appréciation et à l'évaluation du travail des auteurs, éditeurs, imprimeurs, libraires. L'intérêt que peuvent y trouver ces spécialistes peut être, par exemple, de mieux situer les "monuments " plus ou moins connus dans la production totale : Rabelais, Marot, Champier... Ou encore d'avoir une idée plus fine des spécialités de Lyon dans le domaine de l'imprimerie par comparaison avec Paris ou Venise.

Mais la bibliographie doit être conçue pour aider aux travaux des chercheurs qui utilisent eux-mêmes beaucoup le livre de cette époque pour leurs recherches. On pense en particulier aux littéraires, pour qui l'imprimé est une source essentielle, à l'histoire intellectuelle, à l'histoire de la musique et de l'art ; mais cet ensemble de connaissances peut aussi permettre de relancer des études économiques, aider à la constitution d'une biographie intellectuelle... Quantitativement, il est certain que les bibliographes seront bien moins nombreux à utiliser un tel travail que les chercheurs des autres disciplines, si toutefois ces derniers ont connaissance de son existence et si les formulaires de recherche sont adaptés à leurs centres d'intérêt.

\section{Un exemple d'information nécessaire : les pièces liminaires}

Dans mes recherches sur l'histoire de la philologie et l'histoire de l'édition érudite, je me suis aperçue que j'avais besoin, ne serait-ce que pour pouvoir identifier précisément un livre, d'un certain nombre d'informations absentes de la page de titre, en particulier sur les pièces liminaires. Pour identifier simplement une réémission, ou bien une réédition, savoir si elle est corrigée et enrichie ou non, un des outils est de voir s'il y a des modifications dans les épîtres dédicatoires : non seulement le dédicataire, mais le contenu ! En effet, dans la Florence du seizième siècle, un professeur peut dédicacer au même homme, en l'occurrence le grand-duc de Florence, deux éditions différentes du même livre, avec une nouvelle dédicace pour la seconde. Ou bien un imprimeur-libraire peut réunir deux livres différents en un, sans vraiment l'indiquer au titre : là aussi, seule la présence des deux épîtres successives peut l'indiquer au lecteur et lui dire exactement quel est le contenu de ce livre. Il est en effet manifeste qu'une épître dédicatoire est définitivement liée à l'édition qu' elle accompagne, et est systématiquement rééditée en même temps que cette dernière. On connaît ainsi 
nombre de livres précédés de plusieurs épîtres dédicatoires, chacune d'entre elles donnant alors au chercheur des informations précises sur la constitution du livre, la date de la première édition et des rééditions successives...

Autre exemple, celui des multiples éditions du même auteur : j'ai pu en faire l'expérience pour l'imprimeur Sébastien Gryphe, qui à certaines périodes, publie chaque année les mêmes textes de Cicéron, mais offre simultanément plusieurs éditions différentes. Il ne s'agit pas du même livre éternellement réédité, mais de plusieurs éditions : l'éditeur met à la disposition de ses clients, en même temps, des éditions de Melanchthon, d'Estrebay, Vettori, Manuce, Érasme, Hegendorf... Le volume correspondant de la Bibliotheca bibliographica Aureliana ne donne aucune information sur l'éditeur scientifique du livre ; Baudrier, pour sa part, indique parfois à tort que les pièces liminaires sont les mêmes que dans une édition précédente... Donner des informations précises sur les pièces liminaires (auteur, destinataire, date) est donc indispensable pour éviter d'avoir à s'interroger sur des livres qui sont par ailleurs mal localisés, donc pour lesquels on ne connaît pour l'instant que peu d'exemplaires, dans des endroits difficiles d'accès. Le chercheur part parfois sur la piste d'un livre difficilement consultable, dans un local fermé au public, pour s'apercevoir finalement qu'il s'agissait d'une réimpression à l'identique, ou même d'une émission, d'un livre bien plus répandu, information que l'épître dédicatoire aurait pu lui donner...

Il se trouve pourtant que peu de bibliographies incluent dans la description des ouvrages celle des pièces liminaires. Cette réflexion est bien celle de la question du public à qui s'adresse ce travail et de ses besoins. Il est indispensable de ne pas viser prioritairement comme public les concepteurs même de ce travail ; les bibliographes et historiens du livre n'ont pas à être privilégiés dans une entreprise de cette ampleur. Il nous appartient donc d'essayer de sonder les chercheurs de diverses disciplines pour identifier des besoins spécifiques, mieux identifiés par eux peut-être avec l'habitude de l'outil informatique. Autre exemple à prendre certainement en compte : celui des privilèges, parfois imprimés en extrait on in extenso dans les livres, et qui apportent beaucoup d'informations aux historiens du livre mais aussi aux littéraires. 


\section{La localisation des exemplaires connus}

Il me semble qu'une différence importante entre les deux traditions, celles des bibliothécaires et celles des bibliographes, est bien celle de la place accordée aux exemplaires. Pour les bibliographes, la consultation des exemplaires permet d'identifier d'éventuels états différents pour une même édition, ainsi que des émissions, de différencier celles-ci de nouvelles éditions, etc. L'objectif principal des bibliothécaires en revanche a toujours été de décrire et de localiser un exemplaire particulier.

Je crois qu'un des progrès que peut apporter à la bibliographie l'utilisation de l'outil informatique, c'est bien celui de prendre le temps et la place de décrire les exemplaires, comme le fait par exemple VD17 (mais en se restreignant aux bibliothèques participant à ce projet national, c'est-à-dire aux bibliothèques allemandes). Cette description des exemplaires permet dans un premier temps de mieux connaître une édition donnée, et d'identifier les différences qui peuvent se trouver entre les exemplaires d'une même édition. Edit-16 permet par exemple de savoir où se trouvent les états différents d'une même édition, ou les réimpressions proposées la même année (du moins lorsque l’empreinte, c'est à dire le relevé de lettres et d'informations à plusieurs emplacements dans le livre, met en lumière des différences entre des livres qui semblent se rattacher à la même édition); toutefois les exemplaires n’y sont pas décrits. Cette description des exemplaires permet aussi, et plus encore, de s'adresser à un public beaucoup plus large, s'intéressant à l'histoire des collections et des bibliothèques, à l'histoire de la culture et des idées, à la réception des textes...

En réalité le rapprochement entre les deux traditions, particulièrement à propos de la description des exemplaires, a déjà été lancé. Dans l'introduction de la version papier du projet French Vernacular Books, ses responsables annoncent, et ce dès la première phrase, que leur ambition a été « d'identifier tous les exemplaires de toutes les éditions connues des œuvres imprimées dans une langue vernaculaire française $»^{44}$. Et la grille de saisie utilisée pour la constitution de cette base prévoit un nombre important de champs de description pour les exemplaires ${ }^{45}$.

Jean-François Gilmont affiche plus modestement dans GLN15-16 l'objectif de donner les cotes de tous les exemplaires connus. C'est un objectif catalographique : il s'agit de permettre au chercheur qui consulte la bibliographie de savoir où se trouve près de chez lui tel ou tel exemplaire du livre 
qui l'intéresse. La localisation a un intérêt pratique indéniable, en signalant au chercheur tous les exemplaires qui se trouvent relativement près de chez lui ; de telles recherches étaient impossibles en utilisant les bibliographies imprimées, et la complétude des informations permet des recherches plus fines que dans les métacatalogues.

Cet aspect en particulier me permet de rappeler l'objectif du projet BEL16 : à aucun moment, malgré nos longues discussions, la notice n'est devenue centrale et l'objet même - le livre —, secondaire. C'est bien le contraire qui se produit. La bibliographie a pour objectif d'aider à comprendre l'objet livre, à le situer dans son contexte, à avoir sur lui, sur sa conception et sa fabrication, le plus d'informations possibles. Le travail du bibliothécaire et celui du bibliographe se rejoignent ici pour s'arrêter à un moment précis : au moment où vient l'heure de la consultation. Les bibliothèques gardent leurs salles de consultation ouvertes, et assurent à grands frais la conservation de ce patrimoine, en vue de permettre sa consultation actuelle et future. Dans certains cas, la consultation de l'objet livre n'est pas indispensable : la version numérisée apporte alors au chercheur toutes les informations qui lui sont nécessaires, le mode « image » garantissant la fiabilité de la source. Toutefois, la version numérique ne se substitue pas entièrement à l'objet livre, et la tentation de certaines bibliothèques d'interdire systématiquement la consultation des ouvrages montre une méconnaissance parfois étonnante de la particularité du livre artisanal et des travaux de recherche dont il est le support. Elle porte par ailleurs en elle le risque de mettre en danger la préservation du patrimoine écrit, les demandes de budget semblant moins justifiées aux tutelles lorsqu'on ne peut justifier d'un usage de ces collections.

Le travail de recherche pour établir un tel outil — et je dis bien « outil» — s'arrête donc nécessairement au moment où commence le travail d'étude.

\section{Utilisation de ces outils : aspect pratique}

Une dernière remarque s'arrêtera sur les limites d'une bibliographie disponible en ligne. La gratuité semble naturelle dans le cadre d'un projet financé par des institutions publiques; mais la consultation en ligne n'est pas toujours possible. Le bibliothécaire et le bibliographe doivent garder en tête la finalité de ces outils, et leur modalité d'utilisation. En effet, la multiplication des instruments de travail sur Internet rend nécessaire l'adaptation de l'offre de consultation dans 
les salles de lecture où les chercheurs viennent examiner les livres décrits dans les catalogues et les bibliographies. Or, trop de bibliothèques n'offrent encore qu'un accès réduit à Internet, voire même pas d'accès du tout (y compris dans les fonds anciens de certaines grandes bibliothèques universitaires américaines), et le prix de l'abonnement à certaines bases de données payantes met ces dernières hors de portée de nombre d'établissements. Dans certains cas, même si le chercheur peut y avoir accès par son établissement de rattachement (université ou centre de recherche), cet accès n'est disponible que dans les locaux de celui-ci et pas à distance (c'est le cas, en Europe, de EEBO). Il est donc impossible de faire les vérifications nécessaires au moment opportun. Or les habitudes de travail des chercheurs se sont modifiées, et il leur est désormais difficile de travailler sans disposer des outils de travail nécessaires. Les bibliographies sous forme papier gardent donc tout leur intérêt et toute leur utilité pour leurs utilisateurs, et, pour longtemps encore sans doute, devront être mises à disposition dans les salles de lecture des fonds anciens des bibliothèques.

\section{Le travail à mener}

Il est manifeste que, dans l'objectif de donner des informations les plus complètes possibles pour chaque livre, pour chaque imprimeur, le travail est énorme. Nous avons la chance que ce soit un spécialiste qui dirige le projet, et d'autres spécialistes sont prêts à travailler avec nous dès que le projet sera avancé : spécialistes de l'illustration, de la musique imprimée, etc. Même en bénéficiant du travail de nos prédécesseurs, le travail est extrêmement long. Les nouveaux outils disponibles en ligne, qu'il faut patiemment dépouiller, nous font connaître des éditions inconnues des bibliographies déjà publiées. Mais la qualité inégale des catalogues entraîne la nécessité de vérifications poussées. Il ne faut pas se contenter de recopier des notices amicalement fournies par des bibliothécaires. Cela entraîne donc à nouveau une réflexion sur des aspects techniques, dans un mouvement de balancier qui est incessant : il faut envisager de pouvoir disposer d'une grille de saisie facile d'utilisation, qui permette à des correspondants de compléter la bibliographie à distance avec le moins possible de risques d'erreur : saisie guidée, listes déroulantes, validation...

Il faut aussi, d'un point de vue méthodologique, se fixer des étapes, un ordre d'avancement du travail. Comme nos prédécesseurs bibliographes, nous travaillons imprimeur par imprimeur - et non pas localisation par localisation 
ou année par année. Il s’agit donc de donner des descriptions de la production imprimée lyonnaise du seizième siècle, qui, quel que soit le degré de détail que l'on atteindra, seront forcément succinctes, comparées à l'objet livre luimême. L'idée est d'avancer en qualité, à partir de la copie d'une notice, à partir d'une source déjà secondaire mais la plus fiable possible, jusqu’à la description effectuée livre en main, par étapes conduisant à un degré le plus élevé possible de fiabilité et de précision, degré qui sera indiqué dans la notice comme c’est le cas dans GLN15-16.

Le travail a commencé en utilisant pour l'instant le logiciel FilemakerPro qui permet une récupération au format XML ou dans un tableur ; il restera ensuite à compléter les informations secondaires, comme par exemple les références bibliographiques. Il est en cours de transfert sur une plateforme entièrement structurée en XML, e-corpus.

Nous avons donc dès l'origine en tête un projet complexe, constitué de plusieurs bases :

- La base principale décrit les éditions ; l'idéal est de pouvoir rattacher autant de notices d'exemplaire que nécessaire à chaque notice d'édition, qui permettront de décrire aussi bien les différences d'état que les particularités dues à l'histoire de ce livre (provenance, reliure, marques de lecture...);

- Parallèlement, nous prévoyons de déployer une base d'ornements typographiques et de matériel typographique. Ceci est très important car s'il existe des répertoires de caractères utilisés au début du seizième siècle à Lyon, il sera plus facile de les utiliser par un accès en ligne. Notre objectif est bien entendu d'aider à identifier les livres pour lesquels on ne connait pas le nom de l'imprimeur et/ou la date. Nous souhaitons prioritairement pour l'instant étudier la possibilité de réutiliser la base Maguelone, adaptée de la base d'ornements typographiques Passepartout développée à Lausanne par Silvio Corsini et son équipe ${ }^{46}$. Mais le Centre d'études supérieures de la Renaissance de Tours a développé récemment, dans le cadre du programme Bibliothèques virtuelles humanistes, une base de matériel typographique correspondant exactement à nos besoins, $\mathrm{BaTyR}^{47}$;

- Nous avons aussi en projet une base qui mettrait à disposition toutes les reproductions des illustrations présentes dans les livres lyonnais du 
seizième siècle. Nous avons pour l'instant renoncé à ce projet qui dépasse nos capacités humaines et scientifiques.

- Des bases secondaires de références bibliographies et d'autorités seront développées parallèlement.

Qu'en est-il des exemplaires numérisés? Pour l'instant nous n'y avons pas trop réfléchi. Faire des liens vers des livres numérisés est une opération toujours assez longue, car elle se fait manuellement et il faut parfois reconstruire les liens permanents lorsqu'ils existent. Lidéal évidemment serait une recherche qui donne simultanément le résultat dans la base et le résultat dans les bibliothèques numériques : c'est faisable si l'on s'attache à respecter les fonctionnements de l'OAIS, ce qui est le cas d'e-corpus. Un bon nombre de livres lyonnais du seizième siècle ont été numérisés il y a déjà plusieurs années dans Gallica, dont une bonne part à partir d'exemplaires de la bibliothèque municipale de Lyon. Il est sans doute prudent d'attendre la mise en œuvre du projet de numérisation des cinq cent mille volumes du fonds ancien de la bibliothèque municipale de Lyon, confiée en 2008 à la société Google à la suite d'un appel d'offres. Nous pourrons alors, du moins nous l'espérons, travailler directement avec la bibliothèque municipale pour rendre nos projets compatibles et les lier.

Une autre solution, retenue par plusieurs bibliographies en ligne, est d'ajouter aux notices la reproduction de la page de titre (VD17), ainsi que celle du colophon, complément souvent indispensable (Edit-16). Il en est de même pour des projets de bibliographie thématique, comme la bibliographie des éditions juridiques éditées entre le seizième et le dix-huitième siècle, menée au MaxPlanck Institut avec la participation de chercheurs au niveau international ${ }^{48}$. C'est une solution qui permet par ailleurs d'orienter différemment la façon dont sont rédigées les notices : il n'est plus utile de perdre du temps à faire des éditions diplomatiques de la page de titre, soit parce qu'elles existent déjà ailleurs, soit parce que le temps passé à les faire ne se justifie pas par rapport à l'utilisation que pourra en avoir le public, soit parce que la photographie de la page de titre parle mieux qu'un long discours...

Il est de toutes façons indispensable, pour pouvoir mener à bien ce projet qui décrira à terme environ vingt-cinq mille éditions et cent mille exemplaires, d'avoir à nos côtés des spécialistes. Nous prévoyons des collaborations entre projets, pour des raisons techniques, de forces mais aussi de compétence scientifique. Un premier essai à consisté à mettre en ligne une version provisoire 
des éditions d'Étienne Dolet, six mois avant le colloque qui lui a été consacré en novembre 2009 à Lyon. L'objectif était de demander aux chercheurs spécialistes de Dolet, ou bien d'un auteur édité par lui, ou étudiant un livre en particulier, de corriger nos éventuelles erreurs et nous transmettre leurs travaux lorsqu'ils permettaient d'enrichir la bibliographie doletienne. Le résultat a été très encourageant, plusieurs spécialistes ayant contribué à améliorer celle-ci. Espérons que nous pourrons organiser une collaboration internationale autour de ce projet, et qu'après avoir réuni bibliographes et bibliothécaires, nous pourrons bénéficier de l'expertise de littéraires et de chercheurs spécialistes d'histoire religieuse, d'histoire de l'art, d'histoire sociale, économique...

La bibliographie est un domaine qui, sous une apparente simplicité, recèle de multiples difficultés. Concevoir un tel projet demande donc une réflexion poussée sur la méthodologie à mettre en œuvre. Cela demande de considérer les expériences passées, et, dans l'objectif d'assurer l'interopérabilité et la pérennité des données, de prêter une grande attention aux normes et formats existants. Cela demande aussi de prévoir l'utilisation qui pourra être faite de ce travail, afin de le mettre à disposition du plus grand nombre possible d'utilisateurs, sans exclure ceux pour qui sa consultation risquerait d'être rendue difficile par l'emploi de codes spécifiques à un domaine précis. Ce sont les objectifs que nous nous sommes donnés.

Post-scriptum : en juillet 2011, quatre ans après le début du projet, environ douze mille notices ont été établies, couvrant les imprimeurs ayant commencé leur carrière avant 1540. Un test est en cours pour intégrer les notices de BEL16 dans la plateforme e-corpus (http://www.e-corpus.org). Le résultat devrait être disponible en 2012. L'actualité du projet peut être suivie sur le carnet de recherches : http://bel16.hypotheses.org.

\section{Notes}

* Cet article a utilisé largement les réflexions préalables de William Kemp sur le projet : qu'il en soit ici remercié.

1. Henri Baudrier et al., Bibliographie lyonnaise : recherches sur les imprimeurs, libraires, relieurs et fondeurs de lettres de Lyon au XVI siècle (Lyon : L. Brun, 
1895-1921). Voir sur ce projet : Denis Galindo, «Aperçus sur la correspondance de Julien Baudrier, ou l'élaboration de la Bibliographie lyonnaise du XVI siècle ", in Lyon et les livres, dir. Dominique Varry, Histoire et civilisation du livre : revue internationale 2 (2006), p. 229-58.

2. Répertoire bibliographique des livres imprimés en France au seizième siècle (BadenBaden : Koerner, 1968-1978), 20 fascicules ; $2^{\mathrm{e}}$ édition (ibid., 1989-2000), 7 vols.

3. Les volumes couvrant l'édition dans les autres villes avaient en revanche été pour la plupart rédigés par des bibliothécaires français. La Bibliotheca bibliographica Aureliana couvre aussi les dix-septième et dix-huitième siècles. Deux autres ensembles complètent le Répertoire bibliographique pour le seizième siècle : trois volumes consacrés à Strasbourg et sept fascicules, réunis en un volume en 1992, pour la Bibliographie normande, encore incomplète : J. Benzing et J. Muller, Bibliographie strasbourgeoise, 3 vols., 1981-1986 ; Pierre Aquilon, Bibliographie normande, $2^{\mathrm{e}}$ édition, 1992. Voir Jean-François Gilmont, «Lumières et ombres : the French Vernacular Book $\left(15-16^{\text {th }}\right.$ c.) ", Bulletin $d u$ bibliophile no. 1 (2003), p. 149-50.

4. Jean-Dominique Mellot et Élisabeth Queval, avec la participation d'Antoine Mo-naque, Répertoire d'imprimeurs-libraires (vers 1500-vers 1810), 4e éd. (Paris : Bibliothèque nationale de France, 2004) ; Joseph-Marie Quérard, Les supercheries littéraires dévoilées: galerie des écrivains français de toute l'Europe qui se sont déguisés sous des anagrammes, des astéronymes, des cryptonymes, des initialismes, des noms littéraires, des pseudonymes facétieux ou bizarres etc. (Paris : Maisonneuve, 1964, réimpr. de l'édition 1869) ; Antoine-Alexandre Barbier, Dictionnaire des ouvrages anonymes, 4 volumes (Zürich : Hildesheim, 1986, repr. anast. de l'édition Paris, P. Daffis, 1872-1879).

5. http://www.viaf.org/.

6. Alfred Cartier, Bibliographie des éditions des de Tournes, imprimeurs lyonnais (Paris : Éditions des bibliothèques nationales de France, 1937).

7. Claude Longeon, Bibliographie des ouvres d'Étienne Dolet, écrivain, éditeur et imprimeur (Genève : Droz, Travaux d'Humanisme et Renaissance 174, 1980).

8. Verzeichnis der im deutschen Sprachbereich erschienenen Drucke des 16. Jahrhunderts : http://www.vd16.de/ et Das Verzeichnis der im deutschen Sprachraum erschienenen Drucke des 17. Jahrhunderts : http://www.vd17.de/.

9. Censimento nazionale delle edizioni italiane del XVI secolo : http://edit16.iccu.sbn. it/. 
10. La base est hébergée par la ville de Genève : http://www.ville-ge.ch/musinfo/bd/ bge/gln/.

11. http://www.stcv.be/ et http://www.kb.nk/stcn/. Voir sur ces projets et leur histoire Gilmont, p. 148-49. Signalons pour mémoire le Short Title Catalogus Vlaanderen belge (STCV), consacré aux ouvrages publiés au dix-septième siècle.

12. Sur les STC voir plus loin.

13. Voir plus loin.

14. Philippe Renouard, Inventaire chronologique des éditions parisiennes du XVI siècle, d'après les manuscrits de Philippe Renouard, éd. Brigitte Moreau [pour les t. I à IV], 5 volumes parus (Paris : Service des travaux historiques de la Ville de Paris, 1972-). Philippe Renouard, Imprimeurs et libraires parisiens du XVI siècle : ouvrage publié d'après les manuscrits de Philippe Renouard..., 5 volumes parus et 4 fasc. hors-série (Paris : Service des travaux historiques de la Ville de Paris-Bibliothèque nationale, 1964-).

15. Andrew Pettegree, Malcolm Walsby, Alexander Wilkinson, éd., French Vernacular Books: Books Published in the French Language before $1601=$ Livres vernaculaires français : livres imprimés en français avant 1601, 2 vols. (Leiden, Boston : E. J. Brill, 2007).

16. Alexander S. Wilkinson, Books Published in Spanish or Portuguese or on the Iberian Peninsula before 1601, Libros publicados en español o portugués o en la Península Ibérica antes de 1601 (Leiden-Boston : E. J. Brill, 2010) ; Andrew Pettegree, Malcolm Walsby, éd., Netherlandish Books : Books Published in the Low Countries and Dutch Books Printed Abroad before 1601, 2 vols. (Leiden-Boston : E. J. Brill, 2010) ; Id., French Books III \& IV : Books Published in France before 1601 in Latin and Languages other than French, 2 vols. (Leiden-Boston : E. J. Brill, 2011), http:// www.ustc.ac.uk/.

17. http://www.st-andrews.ac.uk/ bookproj/.

18. La conversion rétrospective consiste dans la saisie informatique des fichiers manuels, soit par l'utilisation de notices déjà existantes (notices de la Bibliothèque nationale de France en particulier), soit par la copie des notices manuscrites ou tapuscrites, ce qui entraîne une très grande variété dans leur qualité (dans le cas de fiches établies avant la normalisation de la description, ou bien par des bibliothécaires peu aguerris).

19. Gesamtkatalog der Wiegendrücke, publié par des éditeurs successifs à Berlin, Leipzig, Stuttgart depuis 1925 ; accès internet : http://gesamtkatalogderwiegendrucke.de/ ; ISTC : http://www.bl.ukcatalogue/istc/. 
20. http://www.ubka.uni-karlsruhe.de/kvk/; http://www.worldcat.org/.

21. Rappelons que les objectifs et les modes de constitution des catalogues et des bibliographies sont différents, et parfois opposés. Les premiers ont pour objet la localisation des documents conservés dans une bibliothèque spécifique, la description des livres étant généralement établie à partir de l'exemplaire présent dans la collection.

22. Comme par exemple les répertoires d'autorités déjà cités, les dictionnaires biographiques, les études menées sur les caractères utilisés par les imprimeurs...

23. Gilbert Richard Redgrave, Alfred William Pollard, A Short-title Catalogue of Books Printed in England, Scotland and Ireland, and of English Books Printed Abroad, 1475-1640 (Londres : B. Quaritch, 1926. La réédition mise à jour a été publiée en trois volumes entre 1976 et 1991). Son contenu a été reversé dans Early English Books Online (voir plus loin).

24. Index Aureliensis : catalogus librorum sedecimo saeculo impressorum... (Genève, Baden-Baden, Nieuwkoop : Fondation Index Aureliensis, V. Koerner, De Graaf, 1965-).

25. Chaque volume est consacré aux livres édités dans un pays ou ailleurs dans la langue principale de ce pays : livres en France et en français (1924, rééd. 1966, 1983), en Espagne et en espagnol (1921, rééd. 1989), en Italie et en italien (rééd. 1990), livres allemands et en allemand (1962). Par exemple : H. Thomas, A. F. Johnson et A. G. MacFarlane, Short-title Catalogue of Books Printed in France and of French Books Printed in Other Countries from 1470 to 1600 now in the British Museum (Londres : British Library, 1966 ; réimpr. Londres, 1983 ; Supplement, Londres, 1988 ; Addenda, 1990).

26. Citons par exemple le catalogue des incunables de la Bibliothèque nationale de France, difficile à classer du fait même de la spécificité des incunables (dont beaucoup, par exemple, ne présentent pas de page de titre). Ce catalogue a été lancé dans un souci d'efficacité et d'utilité pour les lecteurs : en effet, alors que le Gesamtkatalog der Wiegendrücke, commencé à la lettre A, avançait lentement, la BnF a entrepris d'éditer à partir 1980 le volume contenant les notices classées par auteur en commençant à la lettre $\mathrm{H}$ pour couvrir dans un premier temps la seconde partie de l'alphabet.

27. On se permet de renvoyer au chapitre "Signaler et faire connaitre les fonds patrimoniaux ", in Manuel du patrimoine en bibliothèque, dir. Raphaële Mouren (Paris : Éd. du Cercle de la Librairie, 2007, collection Bibliothèques), p. 219-64, et 
en particulier les remarques de Yann Sordet sur les priorités que doit identifier le bibliothécaire, p. 235.

28. http://eebo.chadwyck.com/home/.

29. http://estc.bl.uk/.

30. Pettegree, Walsby, Wilkinson, op. cit.

31. Je remercie Malcolm Walsby de m'avoir fourni des saisies écrans de notices complètes de la base.

32. Bien avant les projets électroniques, sur lesquels on reviendra plus loin, c'est la solution retenue par Rodolphe Peter et Jean-François Gilmont, Bibliotheca calviniana : les ouvres de Jean Calvin publiées au XVI siècle, 3 vols. (Genève : Droz, Bibliotheca calviniana 255, 281 et 339, 1991-2000).

33. http://www.sbn.it/.

34. http://www.cheshire3.org/ et http://estc.bl.uk/.

35. Voir Graham Keith Barnett, Histoire des bibliothèques publiques en France de la Révolution à 1939 (Paris : Promodis-Éd. du Cercle de la Librairie, 1987).

36. World Encyclopedia of Library and Information Science, dir. Robert Wedgeworth,

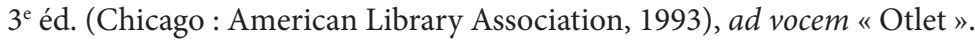

37. International Conference on Cataloguing Principles (Paris : International Federation of Library Associations, 1961). Report (Londres : International Federation of Library Association, 1963), p. 91-96. Également disponible dans : Library Resources and Technical Services, vol. 6 (1962), p. 162-67 ; et Statement of Principles Adopted at the International Conference on Cataloguing Principles, Paris, October, 1961, éd. annotée et commentée par Eva Verona (Londres : IFLA Committee on Cataloguing, 1971).

38. ISBD : International Standard Bibliographic Description. Consolidated edition, edited by the Standing Committee of the Ifla Cataloguing Section (Berlin, Munich : De Gruyter Saur, Ifla Series on Bibliographic Control 44, 2011).

39. Les nouveaux principes sont publiés en ligne sur le site de l'IFLA (International Federation of Library Associations and Institutions) : http://www.ifla.org/files/ cataloguing/icp/icp_2009-fr.pdf/.

40. Utilisé pour le catalogue collectif des bibliothèques françaises, Sudoc, et pour le Catalogue collectif de France, qui permet de mener une recherche simultanément dans le catalogue de la Bibliothèque nationale de France, dans le Sudoc, dans le catalogue rétroconverti des collections patrimoniales des bibliothèques municipales et bibliothèques spécialisées partenaires, ainsi que dans le catalogue 
de plusieurs grandes bibliothèques municipales françaises. http://www.sudoc. abes.fr; http://ccfr.bnf.fr/.

41. Jacques Charles Brunet, Manuel du libraire et de l'amateur de livres..., 3 vols. (Paris : Brunet, 1814) (nombreuses rééditions enrichies). Antoine-Augustin Renouard, Annales de l'imprimerie des Alde, ou Histoire des trois Manuce et de leurs éditions, 3 vol. (Paris : l'auteur, 1803-1812) (plusieurs rééditions enrichies) ; AntoineAugustin Renouard, Annales de l'imprimerie des Estienne, ou Histoire de la famille des Estienne et de ses éditions, 2 vol. (Paris : J. Renouard, 1837-1838).

42. De Florentina Juntarum typographia ejusque censoribus... auctore Angelo Maria Bandinio... (Lucae: typis F. Bonsignori, 1791). Catalogus codicum manuscriptorum bibliothecae Mediceae Laurentianae varia continens opera Graecorum Patrum... (Florentiae : Typis Caesareis, 1764); Catalogus codicum Graecorum bibliothecae Laurentianae, 2 vols. (Florentiae : Typis Regiis, 1768, 1770) ; Catalogus codicum Latinorum bibliothecae Mediceae Laurentianae..., 4 vols. (Florentiae, 1774-1777) ; Catalogus codicum Italicorum bibliothecae Mediceae Laurentianae Gaddianae et Sanctae Crucis... (Florentiae, 1778) ; Bibliotheca Leopoldina Laurentiana..., 3 vols. (Florentiae : 1791-1793). Sur Bandini bibliothécaire voir Emmanuelle Chapron, « Le métier de bibliothécaire au XVIII" siècle : Angelo Maria Bandini à Florence (1726-1803) », Revue d'histoire moderne et contemporaine 51.2 (2004), p. 58-87.

43. http://www.hstl.crhst.cnrs.fr/bibliorome/.

44. Pettegree, Walsby, Wilkinson, p. xix.

45. Je remercie Malcolm Walsby, l'un des responsables du projet, d'avoir mis à ma disposition ces informations.

46. http://maguelone.enssib.fr/.

47. http://www.bvh.univ-tours.fr/, rubrique Iconographie > Matériel d'imprimerie.

48. Census of $16^{\text {th }}$ Century Legal Imprints, sous la direction de Douglas Osler : http:// www.mpier.uni-frankfurt.de/forschungsgebiete/mitarbeiterforschung/oslerbibliography.html/. 